\title{
SVM based fault classification of three phase induction motor
} V.N.Ghate ${ }^{1}$ and S.V.Dudul ${ }^{2}$

${ }^{1}$ Electrical Engineering Department, Government College of Engineering, Amravati (MS), India

${ }^{2}$ Applied Electronics Department, Sant Gadge Baba Amravati University, Amravati, India, vng786@rediffmail.com, dudulsv@rediffmail.com

Abstract: Early detection of abnormal conditions during induction motor's operation would eliminate consequential damages on motor, so that outage time and costs of repairing can be reduced. Due to unique fingerprints from faults in line currents, it is possible to detect faults by extracting fault information from line currents. From the literature review it is observed that in many model based on ANN based techniques, the stator current spectra is used as input feature space. In this paper, simple thirteen statistical parameters are used as an input feature space. Support Vector Machine (SVM) is proposed as a fault classifier. Since the basic SVM is essentially a 2-class classifier, the synergism of three classifiers is proposed to overcome the limitation. Principal Component Analysis (PCA) is used as data fusion method to reduce the dimension of classifier. To verify the performance various kernel function as (Radial Basis Function (RBF), Quadratic, Linear, Polynomial, Multilayer Perceptron (MLP)) are applied and tested with real experimental datasets. In order to generate the experimental data, specially designed $2 \mathrm{HP}$, three phase, 4 pole, $415 \mathrm{~V}, 50 \mathrm{~Hz}$ induction motor is used.

Keywords: Induction Motor, Fault Classification, SVM, PCA.

Introduction

Electrical machines are a necessary part of our daily life. As important elements in electromechanical energy conversion, they are used in many fields, such as power generation, the paper industry, oil fields, manufacturing, etc. Among electrical machines, induction motors, although a complex electro-mechanical devices, are the most widely used in industry because of their rugged configuration, low cost, and versatility. With their great contributions, induction motors are called the workhorse of industry. However, because of natural aging processes and other factors in practical applications, induction motors are subject to various faults. Those faults disturb the safe operation of motors, threaten normal manufacturing, and can result in substantial cost penalties. Such faults can became catastrophic and cause production shutdowns, personal injuries, and waste of raw material. However, induction motor faults can be detected in an initial stage in order to prevent the complete failure of an induction motor and unexpected production costs. Fault detection is the decision if a fault is present or not while fault diagnosis provides more information about the nature or localization of the failure. This certainly reduces downtime expense and reduces the occurrence of catastrophic failures. In order to keep machines in good condition, techniques such as fault monitoring, detection, classification, and diagnosis have become increasingly essential (Isermann, 1997; Jarmo
Ilonen \& Joni-Kristian Kamarainen,2005;Tian Han \& BoSuk Yang, 2006). Invasive and noninvasive methods are reported for machine fault detection (Chow et al.,1993; Filippetti et al., 2000; Singh \& Al Kazzaz, 2003; Onel \& Hachemi Benbouzid, 2008). The noninvasive methods are preferred to the invasive methods because they are based on easily accessible and inexpensive measurements to diagnose the machine conditions without disintegrating the machine structure.

In this paper, an attempt is made to propose the more suitable fault classifier based on Support Vector Machine (SVM). An important feature of the classifier is its capability at generalization: given several different input vectors, all belonging to the same class, the classifier will learn to key off of significant similarities in the input vectors, on the contrary the irrelevant data will be ignored. Therefore the classifier will be applicable to every induction machine, or at least to a sizeable range of induction machines. This research work is based on real time data and so proposed SVM based classifier demonstrates the actual feasibility in a real industrial situation. SVM based classifier presented in this paper has been tested with with different data partitions and about $100 \%$ classification accuracy is achieved.

\section{Feature extraction}

The most common faults, namely stator winding interturn short (I), rotor dynamic eccentricity $(E)$ and both of these (B) are considered. For experimentation and data generation the specially designed $2 \mathrm{HP}$, three phase, 4 pole, $415 \mathrm{~V}, 50 \mathrm{~Hz}$ induction motor is selected. The load of the motor was changed by adjusting the spring balance and belt. Three AC current probes were used to measure the stator current signals for testing the fault diagnosis system. The maximum frequency of used signal was 5 $\mathrm{kHz}$ and the number of sampled data was 2500 .

The main problems facing the use of SVM are the selection of the best inputs and how to choose the classifier parameters making the structure compact, and creating highly accurate classifier. For the proposed system, the feature selection is also an important process since there are many features after feature extraction.

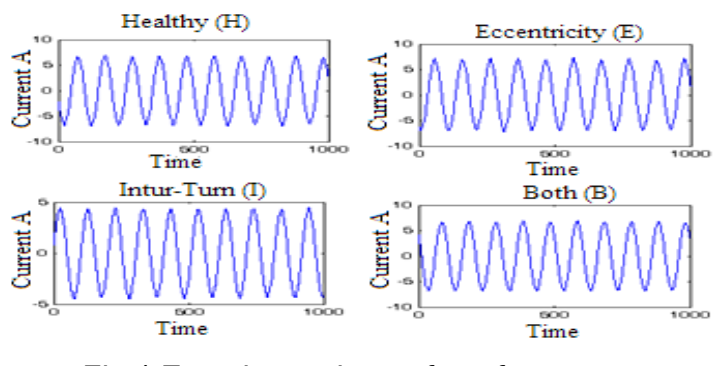

Fig. 1. Experimental waveform for current of phase $A$ for various conditions
Research article

Clndian Society for Education and Environment (iSee)
"Induction motor" http://www.indjst.org
Ghate \& Dudul Indian J.Sci.Technol. 
Many input features need significant computational efforts to calculate, and maybe result in a low success rate. From the time waveforms, as shown in Fig.1, no conspicuous difference exists among the different conditions.

There is a need to come up with a feature extraction method to classify faults. In order to classify the different faults, the statistical parameters are used. To be precise, 'sample' statistics will be calculated for current data. Overall thirteen parameters are calculated as input feature space. Minimum set of statistics to be examined includes the root mean square (RMS) of the zero mean signal (which is the standard deviation), the maximum, and minimum values the skewness coefficient and kurtosis coefficient. Pearson's coefficient of skewness, $g_{2}$ is defined by:

$$
g_{2}=\frac{3(\bar{x}-\tilde{x})}{S_{x}}
$$

Where $\bar{x}$ denotes mean, $\tilde{x}$ denotes median and $S_{x}$ denotes the sample standard deviation. The sample coefficient of variation $v_{x}$ is defined by;

$$
v_{x}=\frac{S_{x}}{\bar{x}}
$$

The $r^{\text {th }}$ sample moment about the sample mean for a data set is given by;

$$
m_{r}=\frac{\sum_{i=1}^{n}\left(x_{i}-\bar{x}\right)^{r}}{n}
$$

$m_{2}$ denotes spread about the center, $m_{3}$ refers to skewness about the center; $\mathrm{m}_{4}$ denotes how much data is massed at the center. Second, third and fourth moments are used to define the sample coefficient of skewness, $g_{3}$ and the sample coefficient of kurtosis, $g_{4}$ as follows:

$$
\begin{aligned}
& g_{3}=\frac{m_{3}}{\left(\sqrt{m_{2}}\right)^{3}} \\
& g_{4}=\frac{m_{4}}{\left(\sqrt{m_{2}}\right)^{4}}
\end{aligned}
$$

The sample covariance between dimensions $j$ and $k$ is defined as;

$c_{j k}=\frac{\sum_{i=1}^{n}\left(x_{i j}-\bar{x}_{j}\right)\left(x_{i k}-\bar{x}_{k}\right)}{(n-1)}$

The ordinary correlation coefficient for dimensions $j$ and $k, r_{j k}$ is defined as;

$r_{j k}=\frac{c_{j k}}{S_{j}-S_{k}}$

\section{Support vector machine}

Support vector machines are learning machines that are able to avoid the curse of dimensionality in both computation and generalization. The SVM theory has been dealt thoroughly (Burges, 1998; Cristianini \& Shawe-Taylor, 2000; Vapnik, 2000; Kecman, 2001). In addition to linear classification, SVM can be applied to non-linear classification problems. When applying SVM in nonlinear problems, nonlinear mapping is used to generate the classification features from the original data. The nonlinearly separable data to be classified is mapped onto a high-dimensional feature space, where the data can be linearly classified (Fig.2) (Burges, 1998).

Using a non-linear vector function $\varphi(X)=\left(\varphi_{1}(X), \ldots, \varphi_{l}(X)\right)^{T} \quad(l>>n)$ to map the $n-$ dimensional input vector $X$ into the 1 -dimensional feature space, the linear decision function in dual form is given by,

$$
f(X)=\sum_{\text {Support Vectors }} \alpha_{i}^{*} y_{i} \varphi\left(X_{i}\right)^{T} \varphi(X)+b^{*}
$$

Where $\alpha^{*}$ - optimal Lagrange multipliers

$b^{*}$ - Optimal scalar, which determine the position of the separating hyperplane.

$y_{i}$ - associated to $x_{i}$ as $y_{i}=1$ if $x_{i}$ belongs to Class I, $y_{i}=-1$ for Class II.

Working in the high-dimensional feature space enables the expression of complex functions, but it also generates problems. Computational problems occur due to large vectors and the danger of over fitting also exists due to high dimensionality. The latter problem is solved above with application of the maximal margin classifier, and so-called kernels give solution to the first problem.

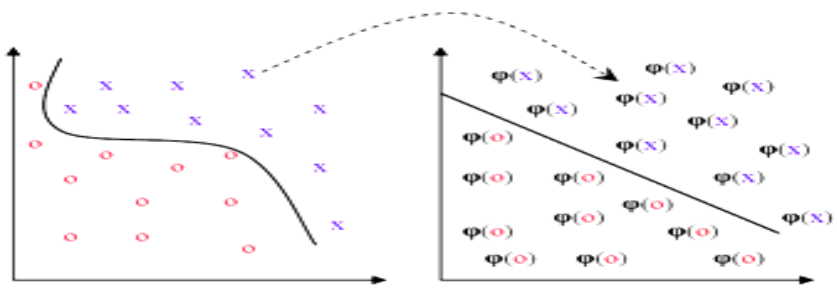

Fig. 2. Mapping the input space to the feature space, where linear classification is possible 


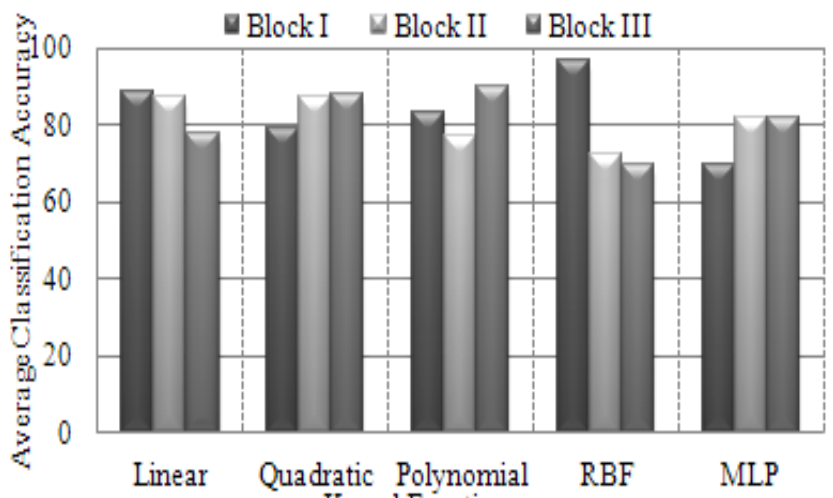

Kemel Functions

Fig.4. Variation of average classification accuracy with Kernel function

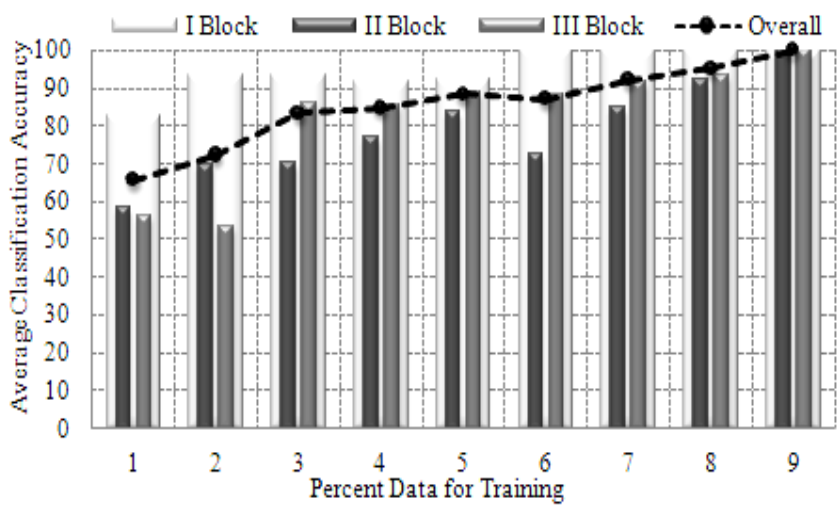

Fig.5. Variation of average classification accuracy with data tagged for training

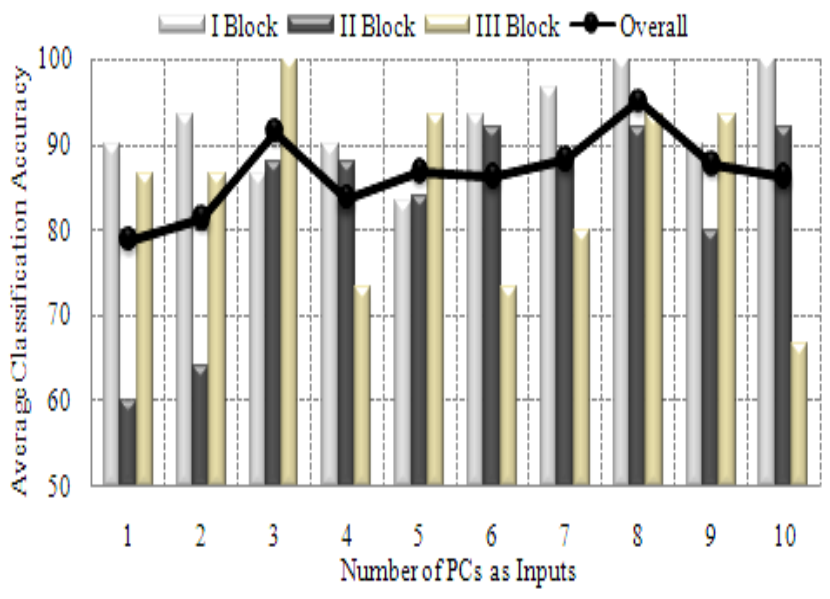

Fig.6. Variation of average classification accuracy with number of $P C s$ as inputs

It is notice that in (8), the data occur only in inner products. A function that returns a dot product of the feature space mappings of original data points is called a kernel $K(x, z)=\varphi(x)^{T} \varphi(z)$. When applying a kernel function, the learning in the feature space does not require explicit evaluation of $\varphi$. Using a kernel function, the decision function will be:

$$
f(X)=\sum_{\text {Support Vectors }} \alpha_{i}^{*} y_{i} K\left(x_{i}, x\right)
$$

and the unknown data example is classified. The values of $K\left(x_{i}, x_{j}\right)$ over all training samples, $i, j=1, ., M$, form the kernel matrix, which is a central structure in the kernel theory. Mercer's theorem states that any symmetric positive definite matrix can be regarded as a kernel matrix that is an inner product matrix in some space (Singh \& Kazzaz, 2003).

Design of SVM for a classification task consists of selection of kernel function which has influence on the decision boundary. In this paper various kernel functions, given as follows are tested, for which MATLAB $2008 \mathrm{R}$ software is used

$$
\begin{aligned}
& \varphi=\exp \left(-\gamma\left|x_{i}-x_{j}\right|^{2}\right) \quad \text {-RBF } \\
& \varphi=x_{i} * x_{j} \quad \text {-Linear } \\
& \varphi=\left(\gamma x_{i} x_{j}+\text { coefficient }\right)^{\operatorname{deg} r e e} \quad \text {-Polynomial } \\
& \varphi=\tanh \left(p_{1} x_{i} x_{j}+p_{2}\right) \quad p_{1}>0, p_{2}<0 \quad-\mathrm{MLP}
\end{aligned}
$$

Support vector machine for fault classification

SVMs are essentially binary classifiers. They are designed to separate only two classes from each other. However, in induction motor fault classification, multiclass classification is required because there exist several fault classes in addition to healthy condition. A solution is to reformulate a multi-class problem to adequate 2-class problems, train classifiers to solve these problems, and then couple the classifiers to reconstruct the solution of the multi-class problem from outputs of the individual classifiers. One of the simplest multi-class classification structures is as shown in Fig.3.

Total data which includes thirteen input features is divided in three groups, first group having two classes Healthy $(H)$ and other faults $(E, I, B)$, second group consist of two classes Eccentricity $(E)$ and other faults (I, $B)$ and third group consist of two classes Inter turn(I) and Both (B). For each block all the kernel functions are verified and average classification accuracy is checked. In each case, ten number of runs are performed for training. Results are as shown in Fig.4.

From results, it is observed that for the first block RBF, for second block Quadratic and for third block Polynomial kernel function gives the optimum results. Classification accuracy is also influenced by the percentage of data tagged for the training and testing. Fig.5 shows the variation of classification average classification accuracy of each block and overall accuracy. In each case five numbers of runs are performed for proper learning and generalization.

PCA for dimension reduction

Principal Component Analysis (PCA) is a technique of multivariate statistical analysis that can linearly or nonlinearly transform an original set of variables into a substantially smaller set of variables. It can be viewed as 
a classical method of multivariate statistical analysis for dimensionality reduction. Due to the fact that a small set of uncorrelated or independent variables is much easier to understand and use in further analysis than a larger set of correlated or dependent variables. This technique has been widely applied to virtually every substantive area including cluster analysis, visualization of high dimensionality data, regression, data compression and pattern recognition. Using PCA, number of inputs to the classifier can be significantly reduced. Fig. 6 shows the performance of individual block and overall classification accuracy varies with number of PCs as inputs. It is observed that eight PCs are sufficient to classify the faults of induction motor with reasonable accuracy of $95.11 \%$. Fig.7. shows a typical 2-D plot for a sample case.

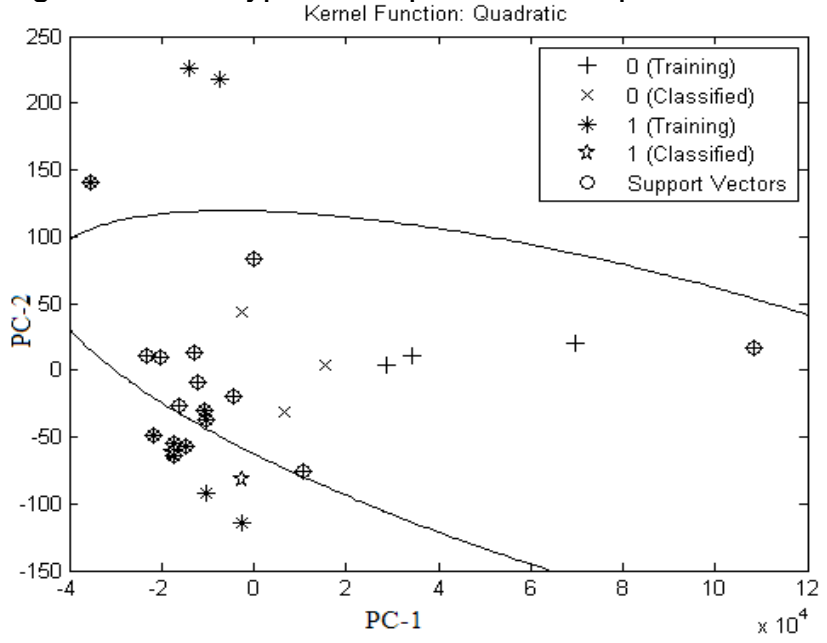

Fig. 7. Typical classification plot for a sample case

\section{Results and discussion}

The SVM based classifier for the fault detection of three phase induction motor is proposed. Since the SVM is essentially a 2-class classifier, the appropriate combination of three classifiers is proposed to overcome the limitation. Each SVM was trained and tested separately with group of dataset with thirteen statistical parameters as feature space. Various kernel functions, namely RBF, Quadratic, Polynomial, Linear and MLP are verified for each SVM and every time they are tested 10 times. It is noticed that RBF for first block, Quadratic for second block and Polynomial for third block gives the optimum results. Also PCA is used as data fusion method to reduce the dimension and it resulted in excellent performance with eight PCs instead of thirteen statistical parameters as inputs. Proposed classifier is carefully tested for various faults and it is found that overall classification accuracy is $95.11 \%$ indicating a reasonable classification.

\section{References}

1. Burges C (1998) A Tutorial on Support Vector Machines for Pattern Recognition. J. data Mining \& Knowledge Discovery. 2(2), 121-167.

2. Chow M-Y, Sharpe RN and Hung JC (1993) On the application and design consideration of artificial neural network fault detectors. IEEE Trans. Ind. Electron. 40, 181-198.

3. Cristianini N and Shawe-Taylo, J (2000) Support Vector Machines and Other Kernel-Based Learning Methods. Cambridge University Press.

4. Filippetti F, Franceschini G, Tassoni $C$ and Vas P (2000) Recent developments of induction motor drives fault diagnosis using $\mathrm{Al}$ techniques. IEEE Trans. Ind. Electron. 47, 994-1004.

5. Isermann R (1997) Supervision, fault-detection and fault-diagnosis methods-An introduction. Control Eng. Practice, 5 (5), 639-652.

6. Jarmo Ilonen and Joni-Kristian Kamarainen (2005) Diagnosis Tool for Motor Condition Monitoring. IEEE Trans. Industry Appln. 41 (4), 963-971.

7. Kecman V (2001) Learning and Soft Computing; Support Vector Machines. Neural Networks and Fuzzy Logic Models. The MIT Press.

8. Onel IY and El Hachemi Benbouzid M (2008) Induction Motor Bearing Failure Detection and Diagnosis: Park and Concordia Transform Approaches Comparative Study. IEEE Trans. Mechatronics. 13, 257-262.

9. Singh GK and Al Kazzaz SAS (2003) Induction machine drive condition monitoring and diagnostic research- a survey. Electric Power Systems Res. 64 (2), 145-158.

10. Tian Han and Bo-Suk Yang (2006) Fault Diagnosis System of Induction Motors Based on Neural Network and Genetic Algorithm Using Stator Current Signals. Hindawi Publ. Corpn. Intnl. J. Rotating Machinery. 1,1-13.

11. Vapnik VN (2000) The Nature of Statistical Learning Theory. Springer-Verlag, New York. 